\title{
Bilateral optic neuritis after COVID vaccination
}

\author{
Valentina Arnao ${ }^{1}$ (D) Mario Baronello Maimone ${ }^{1} \cdot$ Valentina Perini $^{1} \cdot$ Gregorio Lo Giudice $^{2} \cdot$ Salvatore Cottone $^{1}$
}

Received: 24 November 2021 / Accepted: 13 December 2021 / Published online: 31 January 2022

(c) Fondazione Società Italiana di Neurologia 2021

\section{Dear Editor,}

Optic neuritis $(\mathrm{ON})$ has been described in several reports at various stages of COVID-19 infection disease [1]; ON has been observed to be the most common isolated inflammatory syndrome of the central nervous system (CNS) and most frequent serious ocular adverse event following vaccinations, although it has not been reported after COVID vaccination $[2,3]$.

Here, we report the case of a patient, healthy at baseline, who developed a bilateral retrobulbar optic neuritis after exposition to the first dose of Oxford/AstraZeneca ChAdOx1 (AZD1222) vaccine for COVID-19. Neurological complications such as cerebral venous sinus thrombosis (CSVT) due to vaccine-induced immune thrombotic thrombocytopenia [now termed thrombosis with thrombocytopenia syndrome (TTS)], Guillain-Barré syndrome, and peripheral palsy of the VII cranial nerve following adenovector-based COVID19 vaccines have recently been described [4], but other neurological adverse events could be potentially report.

\section{Case report}

The patient was a previously Caucasian healthy middle-aged female with no smoking, alcohol consumption, and medical, malnutrition, or travel history. Two weeks after receiving the first dose of inactivated virus vaccine (Oxford/AstraZeneca ChAdOx1), during mid-April 2021, she experienced headache and painful blurred vision worsened by movement in both eyes, decreased bilateral vision acuity, and no other neurological symptoms. She denied previously contracting COVID-19 or suffering from any of the typical symptoms

Valentina Arnao

arnao.valentina@gmail.com

1 U.O.C. Neurologia con Stroke Unit A.R.N.A.S. CivicoDi Cristina Benfratelli, Palermo, Italy

2 Head and Neck Department, Ophthalmology Clinic, ARNAS CivicoDi Cristina Benfratelli, Palermo, Italy associated with the infection since the beginning of the pandemic. Repeated nasopharyngeal swabs were negative for SARS-CoV-2 on the reverse transcription-polymerase chain reaction (RT-PCR) test during admission in Emergency Room, stay in our Neurological unit care, and at discharge.

Brain CT scan and CT venogram intra- and extra-cranial, carried out in Emergency Room, showed no abnormalities. Neurological examination revealed bilateral loss of vision and decreased color perception, cephalalgia, and no other obvious abnormal findings. On initial ophthalmology examination, she had a visual acuity of $6 / 10$ in both eyes, with ring bilateral scotoma and normal-appearing optic nerves in both eyes without swelling, hemorrhages, or pallor. Optical coherence tomography has observed no abnormalities. Visual evoked potential observed bilateral latency delay (greater on the left eye). Brain and spinal magnetic resonance imaging showed increased signal of the left optic nerve (see Fig. 1). Laboratory tests included blood routine, liver and kidney function, thyroid hormones, tumor markers, C-reactive protein, autoimmune encephalitis antibodies and paraneoplastic antibodies. Serological test for $\mathrm{HBV}, \mathrm{HCV}$, $\mathrm{HSV}, \mathrm{VZV}$, and cytomegalovirus was negative for active infection. Determination of aquaporin 4 (AQP4)-IgG and MOG-IgG has been observed as negative. In cerebrospinal fluid (CSF) leukocyte count, CSF glucose and CSF protein were normal, and no oligoclonal bands were found. Realtime PCR for herpes simplex virus, varicella-zoster, cytomegalovirus and Epstein-Barr virus all resulted negative. Moreover, CSF microscopy and culture were negative for bacteria mycobacterium and fungi. A total body CT scan with contrast and a mammogram have been performed, and no neoplastic lesions and sarcoidosis granulomas have been observed; after treatment with methylprednisolone $(1000 \mathrm{mg}$ for 5 days), the patient's symptoms were greatly improved. Ophthalmologic revaluation with campimetry test and check evoked visual potential reported a full recovery. The patient was then discharged with regular neurology follow-up and the diagnosis was "optic neuritic in the setting of recent Oxford/AstraZeneca ChAdOx1 (AZD1222) COVID-19 vaccination." 


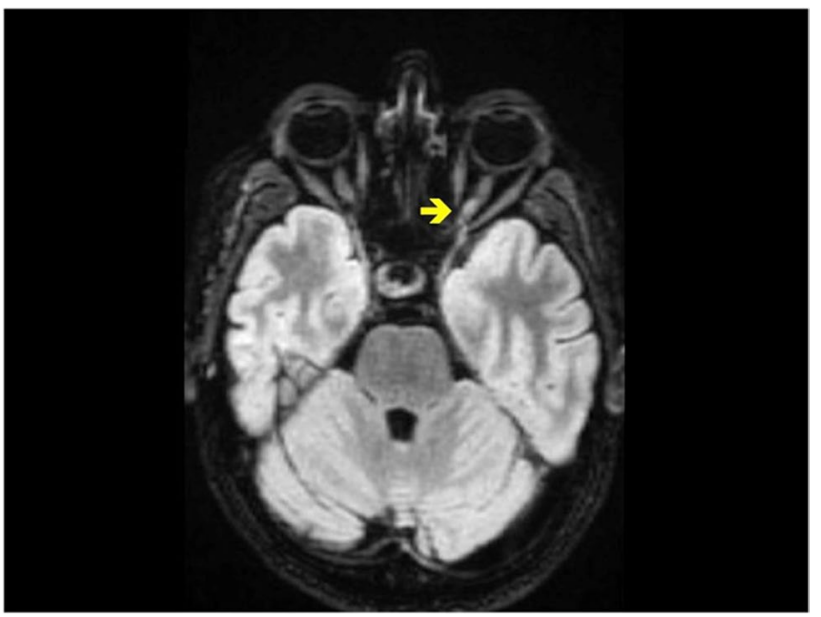

Fig. 1 Magnetic resonance imaging (MRI) of the brain in FLAIR axial showed increased signal of the left optic nerve

This paper aims to highlight the importance of pharmacovigilance and post-marketing surveillance to evaluate the possible correlation between the two events observed. Adverse Drug Reaction Probability Scale has been carried out to highlight causal relationship between an event and exposure to a drug and we have calculated a score of 3 underling a possible relationship [5]. ON is the most frequent serious ocular adverse event following vaccinations against infectious agents [3]. The Vaccine Adverse Events Reporting System (VAERS) is committed to evaluate adverse events potentially but there is no specific surveillance related to ChAdOx1 (AZD1222). EudraVigilance, the European database of suspected drug reaction reports, fosters description of suspected side effects [4].

Barone et al. [6] described two possible patients with ON closely following SARS-CoV-2 mRNA vaccination but a SARS-COV infection complication cannot ruled in at least one case. Despite clear benefits of vaccination for public health, the identification and declaration of potential risks are also imperative. As people underwent to vaccination or booster doses increases, adverse events like $\mathrm{ON}$ are expected to rise, so active surveillance have to be foster.

\section{Declarations}

Ethical approval None.

Conflict of Interest The authors have no conflicts of interest to declare.

Informed consent Written informed consent was obtained from the patient.

\section{References}

1. Sinha A, Dwivedi D, Dwivedi A, Bajaj N (2021) Optic neuritis as a pre- senting symptom of post-COVID-19 multisystem inflammatory syndrome in children (MIS-C). Indian J Pediatr. https://doi. org/10.1007/s12098-021-03921-3 (published Online First: Epub Date)

2. Karussis D, Petrou P (2014) The spectrum of post-vaccination inflammatory CNS demyelinating syndromes. Autoimmun Rev 13:215-224. https://doi.org/10.1016/j.autrev.2013.10.003

3. Cheng JY, Margo CE (2021) Ocular adverse events following vaccination: overview and update. Surv Ophthalmol. 16:S00396257(21)00099-0. https://doi.org/10.1016/j.survophthal.2021.04. 001

4. www.ema.europa.eu/en/medicines/human/EPAR/vaxzevria-previ ously-covid-19-vaccine-astrazeneca\#toverview-section. Accessed on 21 Nov 2021

5. Naranjo CA, Busto U, Sellers EM et al (1981) A method for estimating the probability of adverse drug reactions. Clin Pharmacol Ther 30:239-245

6. Barone V, Camilli F, Crisci M et al (2021) Inflammatory optic neuropathy following SARS-CoV-2 mRNA vaccine: description of two cases. J Neurol Sci 429:118186. https://doi.org/10.1016/j. jns.2021.118186

Publisher's note Springer Nature remains neutral with regard to jurisdictional claims in published maps and institutional affiliations. 